\title{
PERCEPCIÓN DE LA RESPONSABILIDAD SOCIAL EMPRESARIAL EN LOS
} CONSUMIDORES DE SUPERMERCADOS DE LA CIUDAD DE AZOGUES.

\section{PERCEPTION OF CORPORATE SOCIAL RESPONSIBILITY IN THE SUPERMARKET CONSUMERS OF THE CITY OF AZOGUES.}

Jorge Edwin Ormaza Andrade, Mgs. Magíster en Administración de Empresas mención en Recursos Humanos y Marketing (Ecuador).

Docente a tiempo completo de la Universidad Católica de Cuenca sede Azogues, Ecuador jormaza@ucacue.edu.ec.

Juan Diego Ochoa Crespo, Mgs. Magíster en Gestión Empresarial (Ecuador). Docente a tiempo completo de la Universidad Católica de Cuenca, sede Azogues, Ecuador jdochoac@ucacue.edu.ec.

Carlos Fabián Ramírez Valarezo, Mgs. Magíster en Gestión Empresarial (Ecuador). Docente a tiempo completo de la Universidad Católica de Cuenca, sede Azogues,

Ecuador framirezva@ucacue.edu.ec.

Cristian Manuel Ochoa Crespo, Ing. Ingeniero empresarial (Ecuador). Docente de la Universidad Católica de Cuenca, sede Azogues, Ecuador cristian.ochoa@ucacue.edu.ec

\section{ARTÍCULO DE INVESTIGACIÓN}

Recibido: 31 de agosto de 2018.

Aceptado: 30 de noviembre de 2018. 


\section{RESUMEN}

El presente trabajo investigativo indica como las prácticas de Responsabilidad Social Empresarial (RSE) inciden en la percepción de los consumidores cotidianos de los supermercados de la ciudad de Azogues; se mide el grado de influencia directa sobre la calidad percibida, la lealtad a partir de las emociones que las actividades de RSE de estas realizan sobre los consumidores; el estudio pretende demostrar que la inversión en RSE genera diferenciación competitiva mediante la generación de valor para los clientes. La investigación radica que las acciones de RSE ejecutadas por los supermercados no tienen la suficiente claridad en los consumidores tradicionales y por lo tanto, su incidencia al momento de escoger un supermercado para realizar sus compras, puede fortalecerse ostensiblemente por parte del área de marketing de estas empresas. Metodológicamente el abordaje del estudio utilizó el método inductivo; es una investigación descriptiva, transversal, de campo ya que se efectúo una sola medición mediante cuestionarios estructurados dirigidos a los consumidores de los principales supermercados. Resulta interesante estudiar la parte específica de la RSE que ha recibido poca atención, la relativa al comportamiento de los consumidores y sus resultados y poco se conoce sobre la RSE para estos stakeholders y sobre sus percepciones.

Palabras clave: Responsabilidad Social Empresarial, Stakeholders, Consumidores; Marketing.

\section{ABSTRACT}

The present investigative work indicates how the Corporate Social Responsibility (CSR) practices affect the perception of everyday consumers of supermarkets in the city of Azogues; the degree of direct influence on the perceived quality is measured, the loyalty from the emotions that the CSR activities of these perform on the consumers; The study aims to demonstrate that investment in CSR generates competitive differentiation by generating value for customers. The research is that the CSR actions carried out by the supermarkets are not sufficiently clear in the traditional consumers and therefore, their incidence when choosing a supermarket to make their purchases, can be strengthened ostensibly by the marketing area of these Business. Methodologically, the study approach used the inductive method; It is a descriptive, cross-sectional field research since a single 
measurement was carried out through structured questionnaires aimed at the consumers of the main supermarkets. It is interesting to study the specific part of CSR that has received little attention, the relative to the behavior of consumers and their results and little is known about CSR for these stakeholders and their perceptions.

Keywords: Corporate Social Responsibility, Stakeholders, Consumers; Marketing.

\section{INTRODUCCIÓN.}

El análisis histórico de las últimas décadas muestra que las empresas tenían como objetivo esencial el crecimiento y la búsqueda de utilidades, en la actualidad las empresas no deben fijar su horizonte en lo antes descrito. En la actualidad la nueva economía, la cual genera transformaciones significativas en los nuevos procesos económicos y empresariales, la globalización, la era del conocimiento, el saber hacer, la presencia inminente de las tecnologías de la información y la comunicación, la valoración de los activos intangibles manifiestan claramente que es imperante que las empresas se reinventen de manera eficiente, con la idea principal de desarrollar valor para las organizaciones para que sean productivas y competitivas, el presente trabajo investigativo aborda el estudio de la responsabilidad social empresarial a la cual la denominaremos RSE a partir de este momento desde el enfoque de la influencia directa que ésta ejerce sobre el valor percibido por los consumidores y su efecto sobre la intención de compra, satisfacción y lealtad de los mimos. Para la redacción del trabajo investigativo y con el objeto de eliminar redundancias se tomarán como sinónimos las palabras empresa, organización, entidad, a pesar que cada una tiene una conceptualización y significado diferente dentro de las teorías administrativas.

A manera de introducción, los supermercados que comercializan sus diferentes productos en el país y puntualmente en la ciudad de Azogues tienen entre sus políticas diversas actividades de RSE, estas relacionadas con acceso a salud, vivienda y educación, en la actualidad y por mandato de la ley es visible que las organizaciones incluyan la contratación de personas con discapacidades, el cuidado del medio ambiente como la utilización de bolsas biodegradables. Estos son ejemplos de algunas acciones de RSE por parte de los supermercados; ahora bien, se encuentra en discusión si las diferentes acciones de RSE 
ejercidas por los supermercados son desarrollados por filantropía, por fortalecer su imagen, por cumplir con la ley, etc.

En este contexto se ha planteado como objetivo: conocer la influencia directa ejercida por la responsabilidad social empresarial de las actitudes del consumidor y sobre las intenciones de compra.

En concordancia con el objetivo; el estudio desde el enfoque del marketing con orientación directa al stakeholder cliente, analiza una muestra de 365 compradores. El trabajo analiza al consumidor como destinatario final de las actividades de RSE realizada por las organizaciones de la ciudad de Azogues, no se trata de observar el comportamiento del consumidor sino de contrastar en forma real si las acciones de RSE afectan al comportamiento de compra de los consumidores de los supermercados, sus actitudes y sus recomendaciones futuras.

De la formulación del objetivo se desprende la siguiente pregunta de investigación: ¿Influye la Responsabilidad Social Empresarial percibida por el consumidor en las actitudes de los consumidores y sus intenciones de compra?

Del objetivo y la pregunta de investigación antes descrita se formula la siguiente hipótesis; H1: "La Responsabilidad Social Empresarial percibida por los consumidores influye directa y positivamente en las actitudes de los consumidores y en sus intenciones de compra"

Las variables a ser analizadas son:

Variable Dependiente: Y: Responsabilidad Social Empresarial

Variable Independiente: X: Percepción de los consumidores.

Las covariables interrogadas fueron:

$\mathrm{X}_{1}=$ Género del encuestado

$\mathrm{X}_{2}=$ La variable: Comprensión de los consumidores sobre Responsabilidad Social Empresarial 
$X_{3}=$ La variable: Conocimiento si el supermercado de su preferencia realiza acciones sobre Responsabilidad Social Empresarial

$\mathrm{X}_{4}=\mathrm{La}$ variable: Razones fundamentales por las que dejaría de comprar.

$\mathrm{X}_{5}=$ La variable: Acciones de Responsabilidad Social Empresarial

$X_{6}=$ La variable: Disminución de sus compras si su supermercado favorito no realiza actividades de RSE

Para conseguir la concordancia entre objetivo, pregunta de investigación e hipótesis se construye una tabla de congruencias misma que se muestra a continuación:

Tabla 1. Cuadro de Congruencias

\begin{tabular}{|c|c|c|}
\hline OBJETIVO & PREGUNTA & HIPÓTESIS \\
\hline $\begin{array}{l}\text { Conocer la influencia } \\
\text { directa ejercida por la } \\
\text { responsabilidad social } \\
\text { empresarial de las actitudes } \\
\text { del consumidor y sobre las } \\
\text { intenciones de compra. }\end{array}$ & $\begin{array}{l}\text { ¿Influye la Responsabilidad } \\
\text { Social Empresarial } \\
\text { percibida por el consumidor } \\
\text { en las actitudes de los } \\
\text { consumidores y sus } \\
\text { intenciones de compra? }\end{array}$ & $\begin{array}{l}\text { "La Responsabilidad Social } \\
\text { Empresarial percibida por } \\
\text { los consumidores influye } \\
\text { directa y positivamente en } \\
\text { las actitudes de los } \\
\text { consumidores y en sus } \\
\text { intenciones de compra" }\end{array}$ \\
\hline
\end{tabular}

Fuente: Elaboración propia.

Para cumplir con el objetivo planteado, dar respuesta a la pregunta de investigación, rechazar o aceptar la hipótesis formulada en primer lugar realizamos una revisión del marco teórico o estudio del arte que permite enmarcar las principales variables involucradas en el proceso RSE desde la perspectiva del consumidor. A manera de historia y con el propósito de tener un acercamiento significativo al tema, se estructura como parte del documento algunas concepciones elementales sobre diferentes autores para profundizar aún más sobre el constructo responsabilidad social empresarial. En la actualidad se observa permanentemente la inquietud de las organizaciones, ciudadanos y consumidores, por la gestión enmarcada en las concepciones de responsabilidad social empresarial; igual preocupación se traslada hacia el sector público. Ante estos acontecimientos, la responsabilidad social empresarial pretende ser un constructo fundamental en la gestión 
empresarial, organizaciones o empresas respetuosas con todos los stakeholders y esencialmente con lo sociedad y su entorno en general.

\section{REVISIÓN TEÓRICA}

Desde el enfoque de análisis teórico o estudio del arte sobre responsabilidad social empresarial; en la década de los 50 y 60 para Bowen (1953) la responsabilidad social del empresario, radica en que las obligaciones de los empresarios, para que establezcan políticas, tomar decisiones para seguir las líneas de acción que son deseables en términos de los objetivos y valores de la sociedad. Para Davis (1960) manifiesta que algunas decisiones empresariales socialmente responsables pueden justificarse por el beneficio económico a largo plazo de la empresa, pagando de nuevo por su comportamiento socialmente responsable. Bajo esta misma perspectiva Frederick (1960) dice que la aportación privada de los recursos económicos y humanos de la sociedad y una voluntad por parte de las empresas para ver que estos recursos fueron utilizados para fines sociales en general.

Las proposiciones dadas por los autores antes citados, implica una nueva forma de pensar en contraste con la visión de generar riqueza y utilidades, es imprescindible estudiar y resolver los problemas empresariales no como procesos aislados, sino solucionarlos desde la perspectiva de empresas socialmente responsables; vinculados hacia los valores de la sociedad.

Para Johnson (1971) la responsabilidad social empresarial bajo el enfoque del stakeholder aporta con un concepto de mucha significancia para el trabajo investigativo, manifestando que en lugar de esforzarse por conseguir mayores rendimientos para sus accionistas, una empresa responsable tiene en cuenta los intereses de los empleados, proveedores, distribuidores, comunidades locales y la nación en su conjunto. En concordancia directa con el autor la responsabilidad social empresarial no debe ser vista y analizada de manera unidireccional o lineal (accionistas o la obtención de rentabilidad a corto plazo), debe ser considerada de manera holística.

Por otra parte, Carroll (1979) aporta con el concepto sobre el modelo de las tres dimensiones al manifestar que el concepto consiste en responsabilidades corporativas (es decir, económica, legal, ética y filantrópica), las cuestiones sociales de la empresa (por 
ejemplo, las normas laborales, derechos humanos, protección del medio ambiente y lucha contra la corrupción) y acciones corporativas (por ejemplo, reactiva, defensiva, acomodaticia y proactiva). La justificación del presente estudio radica en que los avances que se realicen dentro de esta línea de investigación para profundizar en el impacto de las acciones de RSE en la gestión empresarial, apuntalarán a estimular el proceso de acogimiento de la RSE como parte primordial de la creación de valor para las organizaciones.

En el año 2010 en la norma ISO 26000 sobre el concepto partes interesadas se considera como pilar fundamental la contribución al Seven Core de la responsabilidad social: gobernabilidad, derechos humanos, prácticas laborales, medio ambiente, prácticas justas de operación, asunto de los consumidores y desarrollo e involucramiento de la comunidad. Bajo esta concepción es necesario precisar que explícitamente el aporte de la norma ISO 26000 resulta importante para la comunidad científica; el Seven Core constituye una base fundamental para el desarrollo del trabajo investigativo al referirse en forma precisa a los consumidores entre uno de sus componentes.

En acápites anteriores se hizo referencia con un breve resumen de la evolución de la descripción de la responsabilidad social empresarial, a partir de ahora se desarrollará un recorrido de la evolución del concepto de responsabilidad social empresarial a partir del año 2000.

Los autores McWiliams y Siegel (2001) al conceptualizar la responsabilidad social empresarial dicen que las acciones parecen promover algún bien social, más allá de los intereses de la empresa y de lo que exige la legislación. Marín, Rubio y Aragón (2003) al referirse a la responsabilidad social empresarial manifiestan que es la forma en la que la empresa afronta sus obligaciones de tipo económico, legal, ético o discrecional en beneficio de todos los grupos de interés. Fernández de Gatta (2004) escribe: La responsabilidad social se refiere al proceso mediante el cual las empresas deciden voluntariamente el logro de una sociedad mejor y de un medio ambiente más limpio. Nieto (2004) aporta al explicar que la responsabilidad social es un concepto por el cual las empresas incorporan voluntariamente criterios sociales y medioambientales en las actividades económicas y en las relaciones con sus grupos de interés. Castellano y Chércoles (2005) La responsabilidad 
social nace del propio concepto de ser humano y está ligada a la necesidad que hay en las personas de dignidad para nuestros semejantes y de libertad responsable; donde ser responsables implicaría participar en un proyecto en el que la libertad y los derechos de cualquier persona sean reconocidos. Martén (2005) La responsabilidad social empresarial surge como expresión de la voluntad de las empresas de actuar responsablemente de acuerdo con los intereses sociales y como fiel reflejo de los valores éticos que se ponen de manifiesto en el respeto a las personas, al medio ambiente y a las reglas del juego de la sociedad. Gallizo (2006) La responsabilidad social se refiere a la puesta en práctica de políticas formales y de sistemas de gestión en el campo social y medioambiental, de las que luego debe informar de modo transparente y con contraste externo de los resultados. Cajiga (2009) La responsabilidad social es el compromiso consciente y congruente de cumplir íntegramente con la finalidad de la empresa, tanto en lo interno como en lo externo, considerando las expectativas económicas, sociales y ambientales, de todos sus participantes; demostrando respecto por la gente, los valores éticos, la comunidad, el medio ambiente, contribuyendo así a la construcción del bien común. Huerta, Rivera y Torres (2010) aportan al manifestar que la RSE es una expresión de la voluntad de las empresas a dar cumplimiento no sólo de sus obligaciones con los accionistas, sino también a sus grupos de interés.

Explícitamente los autores emplean una demostración expositiva y convergen en tiempo y en espacio que las acciones que realicen las empresas deben priorizar el beneficio de los grupos de interés y de la sociedad, esta representación de los autores emplean una contrastación racionalista al explicar en sus definiciones que las entidades no deben buscar el beneficio en utilidades a corto plazo, que la gestión de las empresas debe estudiarse desde el enfoque de la interacción con el medio ambiente, los valores éticos, la comunidad contribuyendo a la construcción del bien común; y que la RSE es un compromiso con la comunidad donde se desenvuelve la empresa.

Ahora bien, luego de este transitorio recorrido sobre las conceptualizaciones de responsabilidad social empresarial desde el punto de vista de algunos autores en épocas diferentes; el trabajo se centrará en la relación existente con el stakeholder cliente. Los clientes, consumidores, compradores que se han venido utilizando como sinónimo son la razón de ser de las organizaciones, y alrededor de ellos se debe generar la gestión 
empresarial de las organizaciones. Las empresas con perspectiva de responsabilidad social deben conseguir la mayor combinación de las necesidades, deseos y expectativas de los consumidores como elemento fundamental para el funcionamiento óptimo y adecuado de las empresas. Dicho de otra manera, las empresas deben engranar los requerimientos de la sociedad con los comerciales; con visión sustancial el cliente.

Ante la necesidad de indagar aún más sobre las nuevas aportaciones de la Responsabilidad Social Empresarial y su incidencia sobre el comportamiento del consumidor, se toma con base conceptual la establecida por la Organización Internacional para la Estandarización (ISO) en su norma ISO 26000 está identifica siete materias fundamentales de responsabilidad social que toda organización debería considerar en su estrategia de integración de la responsabilidad social; estas son: Gobernanza de la organización (todo debe, en principio, apoyarse en los criterios y procesos de gobierno de la misma); derechos humanos; prácticas laborales; medio ambiente; prácticas justas de operación (referidas a corrupción, competencia, cadena de valor, etc.); asuntos de consumidores; y participación activa en la comunidad y desarrollo de la misma. Estas materias son, de algún modo, los ámbitos en los que la organización debe centrar su atención a la hora de tratar de actuar responsablemente. Dentro de estos aspectos antes citados el enfoque estará dirigido a uno de ellos por considerar de incidencia directa para el estudio como es el análogo con el "asunto de consumidores".

"La ISO 26000 destaca la importancia de que la organización asuma ciertas responsabilidades con sus consumidores, brindándoles educación e información veraz sobre las estrategias de marketing y contratación, fomentando el consumo responsable y sostenible, y elaborando bienes y prestando servicios que estén al alcance de todas las personas, incluyendo las más vulnerables, entre otras posibles acciones que toda organización debería considerar. La Guía también recomienda tener la responsabilidad de retirar productos del mercado que puedan ocasionar daños, y en caso de que se manejen datos privados, asegurarse de que se mantiene la privacidad de los consumidores. En todo caso, resulta recomendable que la organización tenga en cuenta las directrices de las Naciones Unidas para la protección del consumidor." 
En la misma línea, se puede aseverar que las acciones de responsabilidad social independientemente de que puedan o no tener impacto en el resultado financiero de las empresas, desde el marketing, se han hecho estudios que muestran que avisos publicitarios con elementos vinculados con causas sociales, tienen mayor impacto que aquellos que no utilizan en la comunicación estos elementos. Se debe precisar que el impacto es mayor con consumidores con una mayor conciencia de marca (Nan y Heo, 2007), es decir si la marca ya está presente en la mente de los consumidores, lo que podría llevar a pensar que para una marca ya establecida, invertir en RSE puede resultar más provechoso que para una que no lo está aún.

En la misma perspectiva, para los autores Kuhn y Deetz (2008) definen al consumidor socialmente responsables como el ciudadano que no solo puede satisfacer sus necesidades, sino que las integra en sus decisiones de compra su preocupación por el medio ambiente y otras causas sociales. En palabras de los autores Lozano, Albareda y Arenas (2007) declaran que hay muy poca esperanza de que las mejoras en el medio ambiente se tomen en serio, a menos de que haya un cambio ideológico. Basado en lo anterior es posible inferir que en la medida en la que los diversos actores sociales, entre ellos los consumidores, se involucren en las prácticas de responsabilidad social corporativa, ésta se podrá ir dirigiendo para que mejore tanto lo social como lo medioambiental.

Gonzalez, Korchia, Menuet, Urbain (2009) concluyeron que los consumidores socialmente responsables no forman un grupo homogéneo; por tanto, es posible hacer segmentaciones de tal forma que las compañías orienten sus ofertas a este tipo de segmentos, haciendo referencia al consumo socialmente responsable. En sus hallazgos encontraron cuatro tipos distintos de consumidores: 1) los consumidores socialmente responsables (SCR) que son muy críticos en cuanto al consumo y están muy comprometidos con este tema; 2) los consumidores locales (locals) quienes progresivamente están más preocupados por los efectos negativos del consumo, especialmente en el tema de la basura, y específicamente en su entorno inmediato; 3 ) los consumidores buena causa (good causes) que asocian el consumo con el placer aunque son conscientes del efecto pernicioso en el sistema, y se orientan a modos alternativos como el comercio justo y a las prácticas de desarrollo sustentable; y finalmente, 4) los consumidores indiferentes (indifferent) que muestran un punto de vista del consumo altamente microsocial y microindividual, para quienes el 
consumo socialmente responsable no forma parte de sus intereses. Tomando como referencia estos hallazgos, se puede inferir que un consumidor crítico y comprometido con la responsabilidad social tenderá a ser más exigente en cuanto a la presión con la que demandan estas causas a las empresas.

Con la exigencia cada vez mayor por parte de los consumidores sobre productos verdes, que las empresas implementen valores, ética, consciencia social incita a que las empresas incorporen acciones de responsabilidad social empresarial (RSE).

\subsection{Stakeholders o Grupos de Interés.}

Por otra parte, es adecuado referirse a la otra variable que servirá de base para el presente documento es la concepción de Stakeholders que se describe como un grupo o individuo que está relacionado o vinculado con las actividades de una empresa. Los principales grupos de interés son: accionistas, empleados, inversionistas, proveedores, consumidores, la sociedad en general, etc. Al respecto Oviedo (2011) dice que "la Teoría de los Stakeholders como modelo de gestión empresarial plantea que se deben considerar las expectativas de los grupos de interés como medio para conseguir los objetivos económicos de la empresa. Asimismo, conceptualiza a la empresa como una institución social que configura un proyecto plural, en el que toman parte un número de grupos muy diversos, con variadas exigencias, por lo cual se debe buscar la participación de todos aquellos, de forma directa o bajo representación, poseen algún tipo de vinculación con los objetivos de la organización"

\section{MATERIALES Y MÉTODOS}

El artículo sobre Responsabilidad Social Empresarial (RSE) hace frente al problema de investigación utilizando el método inductivo, desde un enfoque cuantitativo descriptivo de tipo transversal y de campo. Como población de estudio para el presente trabajo investigativo se toma a los compradores habituales de los principales supermercados ubicados en el cantón Azogues; la capital de la provincia del Cañar tiene un población proyectada de 83.770 habitantes, de acuerdo al censo del año 2010 efectuado por el Instituto Nacional de Estadísticas y Censos (INEC); dentro de esta urbe existen alrededor 
de 15 supermercados de los cuales para el estudio se ha tomado como muestra a seis de ellos como son: AKI, TIA, Centro Comercial Suiza, La Bodega, Comisariato Rosita, Centro Comercial Jerez Shopping es importante señalar que Centro Comercial Suiza, La Bodega, Comisariato Rosita, Jerez Shopping son de inversión de habitantes de la ciudad capital de los Cañaris, las otras dos son marcas de supermercados de venta y comercialización a nivel nacional, la razón fundamental de considerar a estas seis marcas de supermercados radica en el hecho de que son las más visitadas al momento de escoger donde realizar las compras por parte de los consumidores de la ciudad de Azogues. Para el cálculo de la muestra se estimó 7.200 consumidores en el lapso de un mes, que realizan sus compras en los supermercados antes citados, valor obtenido mediante observación directa o visita in situ en los diferentes establecimientos comerciales.

El cálculo de muestra se realizó utilizando la herramienta informática Excel con la fórmula de muestreo aleatorio simple para la cual se estableció un nivel de confianza del 95\%, este valor asegura una muestra de un tamaño representativo con un $95 \%$ de confiabilidad y un error de estimación del $5 \%$. Como se demuestra en el siguiente gráfico:

Gráfico 1. Cálculo de la Muestra

\section{FORMULA DE ESTIMACION DE LA MUESTRA (IDEAL)}

\begin{tabular}{|c|c|c|c|c|c|c|}
\hline & Universo (N) & $\begin{array}{c}\text { Muestra } \\
\text { (n) }\end{array}$ & $\begin{array}{c}\text { Error de } \\
\text { estimación } \\
\text { (e) }\end{array}$ & $\begin{array}{c}\text { Nivel } \\
\text { confianza (o) }\end{array}$ & $\begin{array}{c}\text { Probabilidad } \\
\text { a favor }(p)\end{array}$ & $\begin{array}{l}\text { Probabilidad } \\
\text { en contra (q) }\end{array}$ \\
\hline Total & 7200 & 365 & $5,00 \%$ & 1,960 & $50 \%$ & $50 \%$ \\
\hline & & & & $95 \%$ & & \\
\hline
\end{tabular}

Fuente: Elaboración propia.

Realizado el cálculo se obtiene un tamaño muestral de 365 encuestas dirigidas a consumidores habituales de los principales supermercados de la ciudad; como fuente de información primaria. El propósito específico de la aplicación de los cuestionarios fue conocer la opinión y expectativas de los consumidores en torno a la RSE así como sus actitudes hacia los supermercados y su vinculación con las causas sociales, además de conocer el grado de involucramiento del consumidor. 
Para la obtención de información se recurre a un cuestionario estructurado; antes de su aplicación se desplegó una prueba piloto a 15 consumidores, con el objetivo de determinar la adecuada estructuración, coherencia, orden, lógica de las preguntas; se hicieron siete preguntas que se vinculan directamente con el objetivo del presente artículo y aceptación, rechazo o nulidad de la hipótesis. La información que se obtuvo de la encuesta fue procesada por medio de tablas dinámicas en el paquete utilitario Excel.

\section{ANÁLISIS DE LOS RESULTADOS}

Una vez que con la información fue procesada con la utilización de tablas dinámicas se procedió a realizar la gráfica de las variables e interpretación de las mismas y de esa manera mostrar las implicaciones teóricas y prácticas de la investigación.

Para el estudio se desarrolló inicialmente una clasificación de la muestra por género (covariable $\mathrm{X}_{1}$ ) como se observa en la siguiente tabla 2:

Tabla 2. Clasificación de la muestra por género

\begin{tabular}{|c|c|c|}
\hline GÉNERO & FRECUENCIA & PORCENTAJE \\
\hline MASCULINO & 134 & $37 \%$ \\
\hline FEMENINO & 231 & $63 \%$ \\
\hline TOTAL & 365 & $100 \%$ \\
\hline
\end{tabular}

Fuente: Elaboración propia.

\subsection{Preferencia del Supermercado al momento de realizar sus compras.}

Las personas con respecto al principal supermercado donde efectúan sus compras, la que mayor aceptación obtuvo fue AKÍ con un 27,7\%, luego en orden de aceptación La Bodega 
con un 24,4\%; TíA 15,3\%; Centro Comercial Suiza con un valor de 12,6\%, Comisariato Rosita 10,1\%, y Jerez Shopping 9,9\%.

Gráfico 2. Preferencia del Supermercado

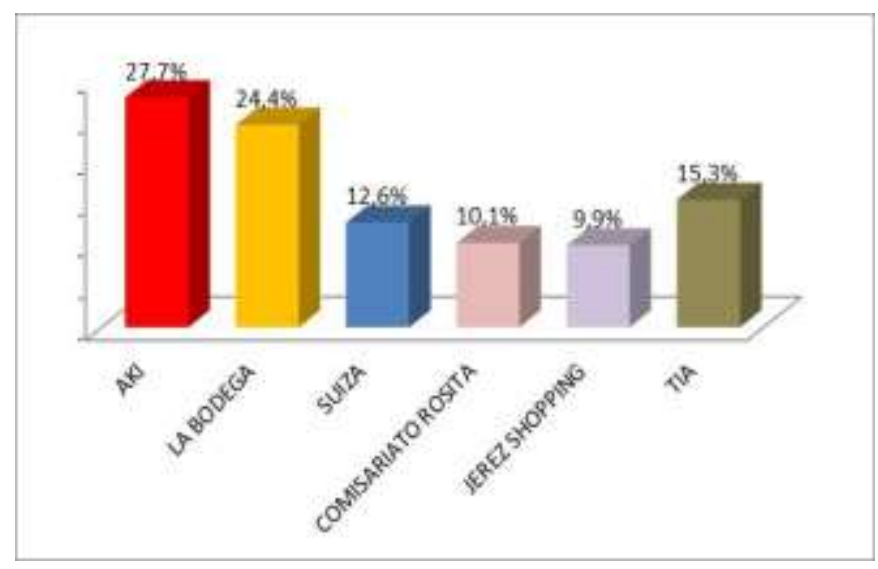

Fuente: Elaboración propia.

\subsection{Razones al momento de realizar sus compras.}

Como motivo principal para optar por un supermercado es el precio con una ponderación de $32,1 \%$, luego se encuentra el servicio que ofrecen los supermercados de la ciudad con un valor del 20\%; surtido de productos con un $18,1 \%$, cercanía o ubicación $17 \%$. Los porcentajes derivados de la pregunta en cuestión refleja de que los consumidores acceden a un supermercado buscando el ahorro, pero vinculado a un buen servicio y surtido de productos restando importancia la ubicación, tradición e infraestructura. 
Gráfico 3. Razón al momento de realizar sus compras

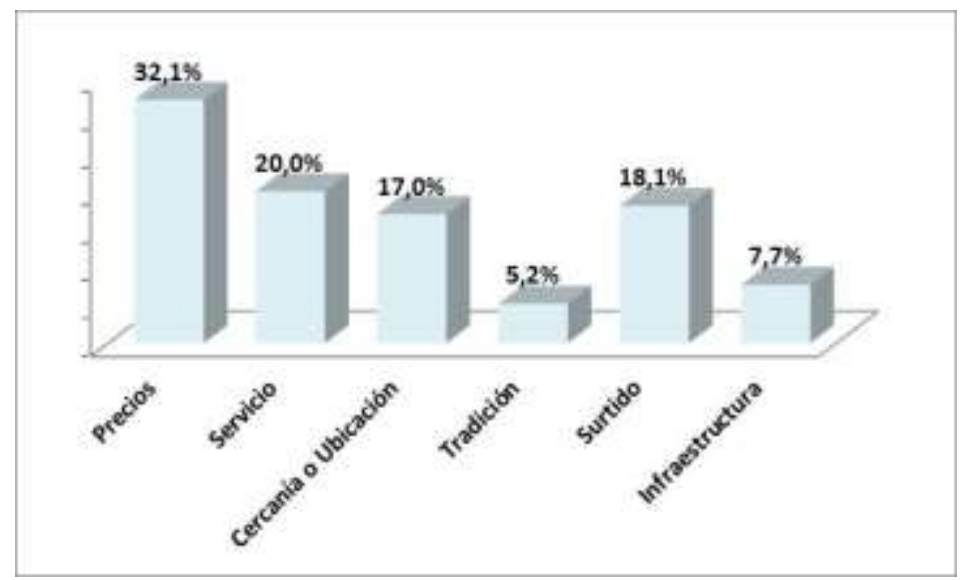

Fuente: Elaboración propia.

\subsection{Comprensión de los consumidores sobre Responsabilidad Social Empresarial}

La covariable $\mathrm{X}_{2}$ corresponde a la pregunta que fue realizada con el propósito esencial de averiguar si las personas encuestadas tienen conocimiento sobre el concepto de RSE; la indagación se realizó sin ningún tipo de información; con la idea de que no exista sesgo al momento de aplicar la misma. De la pregunta se obtuvo un porcentaje alto de encuestados que conocen sobre el término de RSE.

Gráfico 4: Comprensión sobre RSE

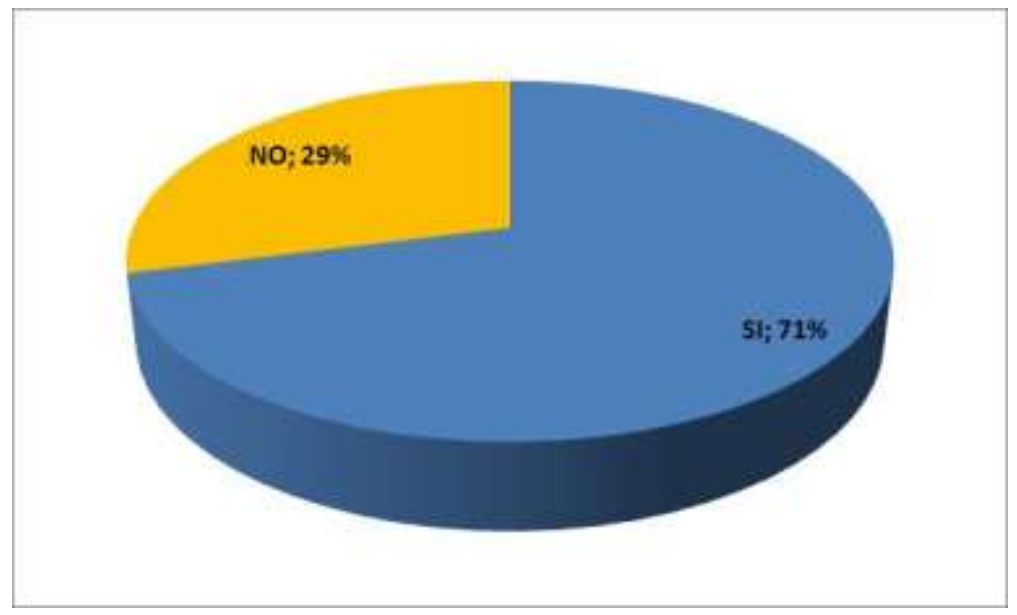

Fuente: Elaboración propia. 


\subsection{Conoce si el supermercado de su preferencia realiza acciones sobre Responsabilidad Social Empresarial}

Los encuestados al responder a la pregunta si el supermercado de su preferencia realizan acciones de RSE correspondiente a la covariable $\mathrm{X}_{3}$; el mayor porcentaje que corresponde al $53 \%$ respondió que no y tan solo el $47 \%$ conoce sobre las actividades de RSE, esta pregunta es muy significativa para el estudio y para los acciones de los supermercados para que se tomen medidas correctivas vinculadas con un adecuado plan de marketing a fin de que se exista comunicación constante y permanente de las actividades de RSE que las empresas desarrollan.

Gráfico 5: Conocimiento sobre acciones de RSE de los supermercados

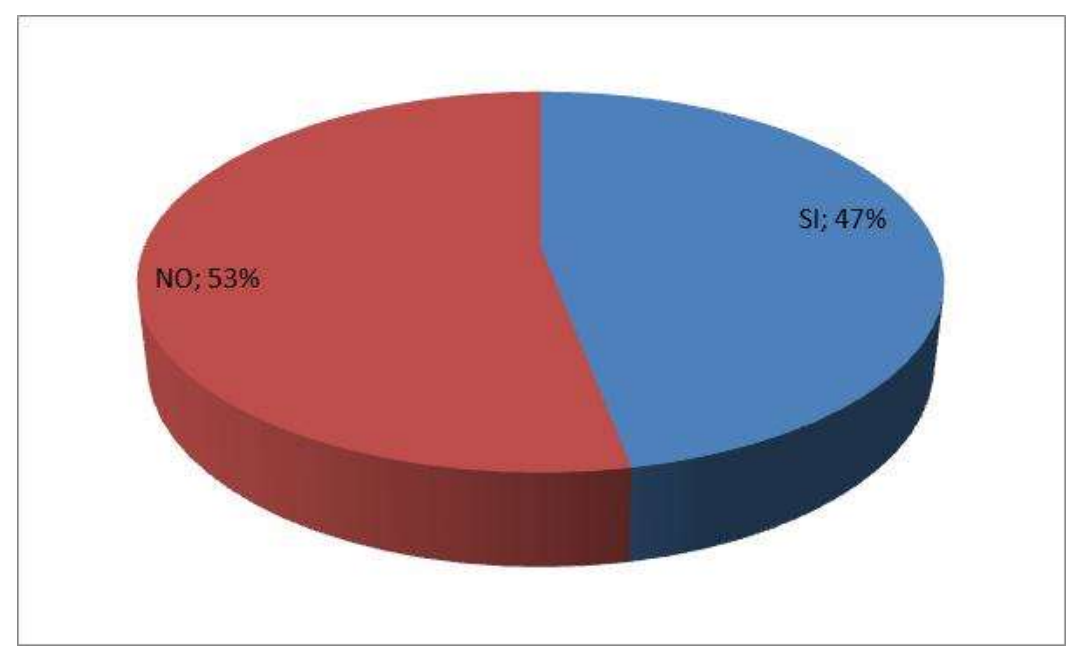

Fuente: Elaboración propia.

\subsection{Razones fundamentales por las que dejaría de comprar.}

De las 365 personas encuestadas que dejarían de comprar en el supermercado de su preferencia (covariable $\mathrm{X}_{4}$ ) atribuyen como razón principal al precio con un 40\%; por recibir un servicio inadecuado $35 \%$, un $20 \%$ por la calidad de los productos, y sólo el $5 \%$ lo haría por falta de acciones de RSE como lo demuestra el siguiente gráfico. 
Gráfico 6. Razones para dejar de comprar.

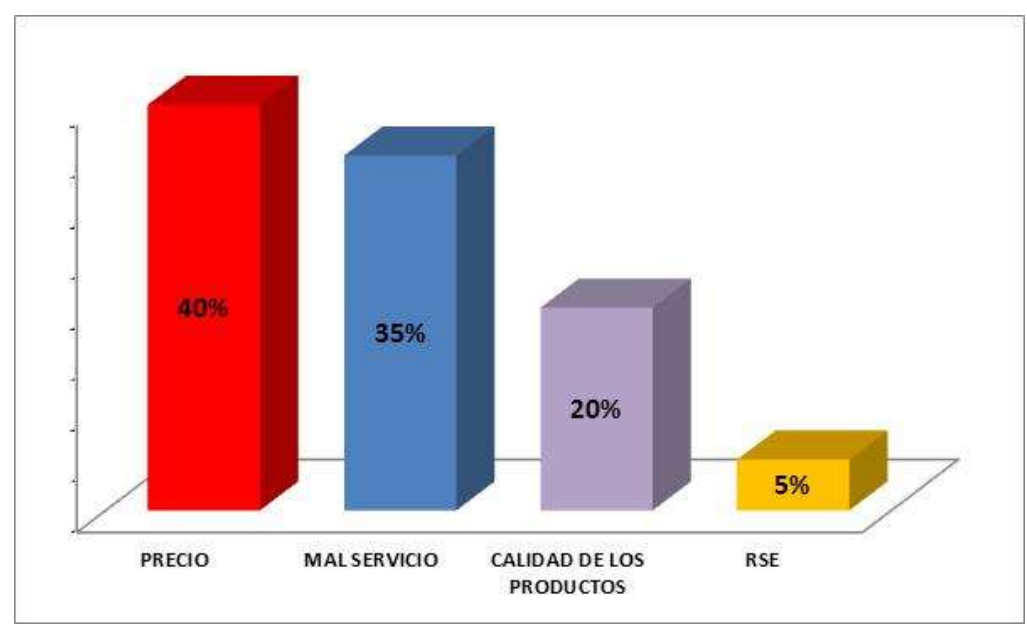

Fuente: Elaboración propia.

\subsection{Acciones de Responsabilidad Social Empresarial}

Los encuestados al ser interrogados sobre las actividades de RSE, que el supermercado de preferencia, realiza en beneficio de la colectividad covariable $X_{5}$; dieron variadas respuestas entre las que se citan las siguientes: cuidado con el medio ambiente (reciclaje, reutilización, reducción), ayuda a sectores vulnerables, campañas de ayuda social; regalías en programas navideños. Es necesario en este punto hacer referencia que algunas respuestas dadas por las personas encuestadas, no se apegan al concepto de RSE; por tanto es imprescindible que las empresas en estudio realicen o incorporen en su gestión empresarial campañas de marketing y estrategias publicitarias sobre sus ejercicios de RSE.

\subsection{Disminuiría sus compras si su supermercado favorito no realiza actividades de RSE}

Al referirnos al hecho de que el supermercado donde los consumidores realizan sus compras en forma habitual covariable $\mathrm{X}_{6}$, reduciría, o dejara de enfocar la responsabilidad social empresarial a algunas de sus gestiones o estrategias empresariales, los compradores optarían por no adquirir los productos, el $41 \%$ contesto de manera positiva y 
el $59 \%$ de manera negativa. Este resultado es muy interesante ya que en un porcentaje mayor no les interesa si la empresa deja o no de contribuir con acciones de RSE. Por lo dicho; el resultado de esta pregunta es valioso por su información, ya que de ella se deriva acciones que las empresas deben desarrollar en relación al mercado potencial o nicho de mercado, realizando acciones de marketing y comunicación para que la RSE sea considerada como ventaja competitiva.

Gráfico 7: Dejaría de comprar si su supermercado no realiza actividades de RSE

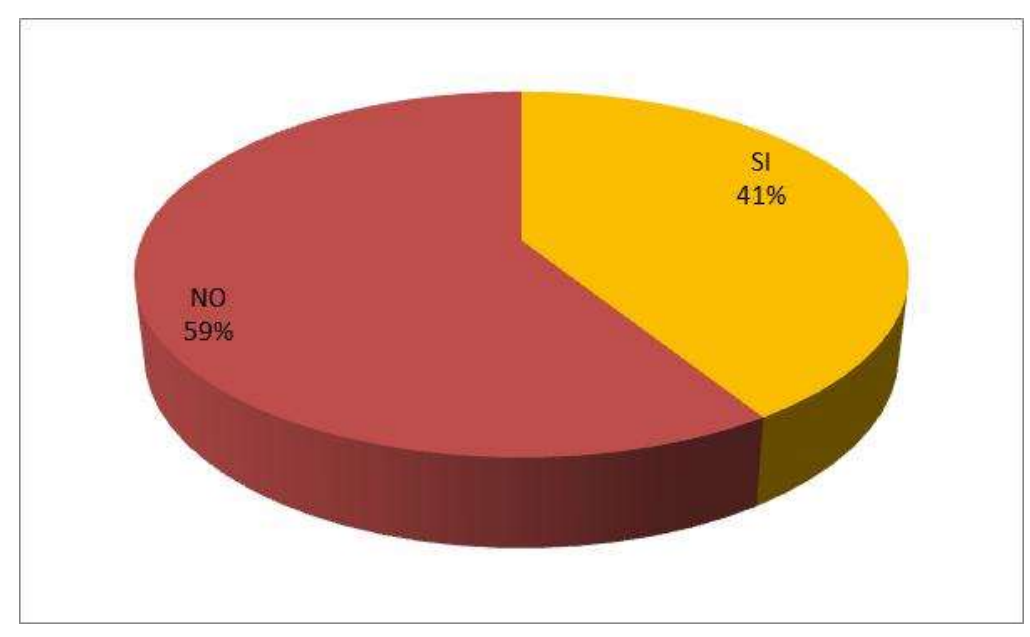

Fuente: Elaboración propia.

\section{CONCLUSIONES}

Las acciones de responsabilidad social empresarial no son concluyentes al escoger un supermercado donde realizar sus compras de manera habitual, el cliente se fija netamente en el precio, el servicio, la ubicación la calidad de los productos.

De acuerdo al estudio desarrollado en los principales supermercados de la ciudad de Azogues, existe un desconocimiento marcado de la concepción sobre responsabilidad social empresarial, no se tiene claro las definiciones como el real significado de la RSE, hay total confusión de actividades que realizan las empresas con los conceptos de RSE, como por ejemplo podemos citar la inclusión de personas con discapacidad en la nómina de las empresas, esto más bien es un mandato de ley antes que una acción de responsabilidad 
social empresarial. Es necesario señalar que existe un pequeño porcentaje que conocen algo de RSE como por ejemplo el cuidado con el medio ambiente, al señalar puntos clave como son: el reciclaje, reutilización y reducción que deben desarrollar las organizaciones como buenas prácticas en su gestión y trabajo empresarial.

Existen actividades que resaltan a la vista y que los consumidores plenamente identifican como acciones de RSE como, por ejemplo; cuidado con el medio ambiente (reciclaje, reutilización, reducción), ayuda a sectores vulnerables, campañas de ayuda social; regalías en programas navideños.

Se evidenció que el término RSE, es todavía un constructo que los consumidores asumen como positivo para el desempeño de las empresas, no es un factor que influye directamente al momento de seleccionar un supermercado donde realizar sus compras en forma habitual.

Es necesario precisar que las organizaciones (supermercados de la ciudad de Azogues) invierten en RSE de manera adecuada, estas no se han comunicado a la razón de ser de las empresas; el stakeholder consumidor; por lo tanto, las empresas deben desarrollar campañas agresivas de marketing y comunicación, con el objetivo de que las buenas prácticas de RSE se conviertan en ventaja competitiva y sean un puntal fundamental para el posicionamiento de las entidades en la mente del consumidor.

Las organizaciones en la actualidad deben reinventar su gestión empresarial construyendo una visión integral dirigida hacia acciones de RSE; llegar a buscar la relación constante de interdependencia entre las empresas y los grupos de interés (consumidores).

En la actualidad, la RSE es un tema de mucho interés para gobiernos, empresas, grupos de interés en vista del daño ambiental que se está ocasionando con el propósito de producir por producir sin ningún control contra la afección que se ocasiona al medio ambiente y por lo tanto a la sociedad. Por lo manifestado las empresas buscan una orientación hacia prácticas de RSE no por mera filantropía y con la vaga idea de convertirse en juntas de beneficencia; sino con la intención de incorporar a su visión empresarial las concepciones, perspectivas de RSE con el ánimo de generar competitividad empresarial y conseguir una rentabilidad a largo plazo.

Al considerar al consumidor y su nuevo comportamiento, así como sus actitudes al momento de realizar sus compras es necesario enfatizar su percepción sobre las 
organizaciones socialmente responsables, por lo tanto, es imprescindible que las empresas conozcan el pensamiento de los consumidores sobre las prácticas de RSE, y los empresarios deben enfocar sus gestiones hacia dicho pensamiento.

Si los empresarios siguen al pie de la letra los enunciados teóricos, los postulados, las definiciones, los conceptos de RSE, se tiene como respuesta una mejor calidad de vida para los stakeholders, por lo tanto, se consigue un desarrollo social y económico. En suma, se puede estimar que las empresas que se preocupen de perfeccionar la apreciación de los compradores obtendrán como resultado satisfacción, fidelidad, incremento de sus ventas, utilidades, mejorar la imagen corporativa, rentabilidad, sostenibilidad.

Como conclusión final, la academia, el gobierno, las organizaciones tanto públicas como privadas, tienen la obligación de desarrollar proyectos para transmitir y consolidar la responsabilidad social empresarial como un eslabón importante para lograr progreso sustentable y sostenible.

\section{REFERENCIAS BIBLIOGRÁFICAS}

Abadía, J. M., \& Macarulla, F. L. (1996). Análisis de la información sobre responsabilidad social en las empresas industriales que cotizan en bolsa. Revista española de Financiación y Contabilidad.

Aguilar -Platas, A. y E. Raufflet (2010). Desviación positiva y responsabilidad social empresarial (RSE). En: Innovar. Revista de Ciencias Administrativas y Sociales, no. 20 (37), 22 -30. Altés, C.

Alvarado, A. (2008). Responsabilidad social empresarial percibida desde una perspectiva sostenicéntrica, y su influencia en la reputación de la empresa y en el comportamiento del turista. PhD diss., Universidad de Valencia.

Alvarado, A. (2007). Marketing y Responsabilidad Social Empresarial: un mapa de navegación. Teoría y Praxis, 4, 118-140. 
Bigné, E., Andreu, L., Chumpitaz, R., \& Swaen, V. (2006). La influencia de la responsabilidad social corporativa en el comportamiento de compra de estudiantes universitarios. Esic Market, 6 (597), 163-189.

Currás, R. (2007). Comunicación de la responsabilidad social corporativa: imagen e identificación con la empresa como antecedentes del comportamiento del consumidor. Tesis doctoral. Universidad de Valencia, España.

Délano, B (2005). Responsabilidad Social Empresarial: Un nuevo campo de gestión donde las Relaciones Públicas tienen un rol. Ecuador. Revista ALACAURP № 4.

Fernández, G., R. (2005). Administración de la responsabilidad social corporativa. Thompson. Madrid, España.

González, M. (2011). El porqué de la Responsabilidad Social Corporativa. Consultado en julio de 2011.

Hernández Sampieri, R., Fernández Collado, C., \& Baptista Lucio, M. (2010). Metodología de la Investigación. México: Mc Graw Hill- Interamericana Editores.

ISO/TMB/WG/SR, 2010. 'Guía de responsabilidad social ISO 26000 (traducción oficial)'. International Organization for Standardization, Génova.

López, Y. (2008). Las últimas tendencias del consumidor en RSE. México.

Martínez, J.L., Carbonell, M. \& Agüero, A. (2006). Los stakeholders y la acción social de la empresa. Madrid: Marcial Pons, ediciones jurídicas y sociales.

Vargas, J. (2005). Responsabilidad Social Empresarial (RSE) desde la perspectiva de los consumidores. Documento de proyecto. CEPAL. Santiago de Chile. Ed. Naciones Unidas. 